\title{
ROLE OF FREE VOLUME IN POLYMER SOLUTION THERMODYNAMICS
}

\author{
D. PatTerson \\ Chemistry Department, McGill University, Montreal, Canada
}

\begin{abstract}
The principal effects determining the value of the free energy of mixing of polymer solutions are discussed and compared, namely the combinatorial, energetic and structural effects. The influence of the last on the thermodynamic behaviour of polymer solutions is then dealt with, as revealed in the lower critical solution temperature, and in the effect of pressure on polymer-solvent compatibility, the second virial coefficient and the coil dimensions. Quantitative aspects of polymer solution thermodynamics are then discussed; arguments in favour of the use of the segment fraction (instead of the volume fraction) in expressing the combinatorial term are given, and the noncombinatorial contribution at zero pressure and the effect of pressure on the $\chi$ parameter are characterized. Finally, a difficulty is noted in assessing the possible effect of a difference in diameter between polymer chains and solvent molecules or chains.
\end{abstract}

The first topic to be dealt with by the Microsymposium is 'interactions arising from the difference in size and shape of the polymer and solvent molecules'. The most important interaction of this type comes from the 'structural effect' of Prigogine, Mathot, Bellemans and Trappeniers ${ }^{1}$. The "equation of state effect' which has become familiar through the recent work of Flory and collaborators ${ }^{2}$ is similar in nature. The importance of the structural effect was recognized twenty years ago by Mathot ${ }^{3}$. It is the main new concept in the theory of polymer solution thermodynamics of Prigogine and collaborators.

\section{THE STRUCTURAL EFFECT}

Introducing the structural effect, Prigogine points out that a polymer liquid and a solvent or 'monomeric' liquid typically have very different equation of state properties. This is related to the polymer having a very low degree of thermal expansion or 'free volume' $\dagger$ compared to the solvent. This difference of free volume is independent of any difference in chemical

$\dagger$ The term 'free volume' is chosen here because of its intuitive appeal in an essentially qualitative discussion. It was not used by Prigogine and collaborators, and may be replaced by terms such as 'degree of thermal expansion above close-packed volume'. 
nature between the polymer and the solvent, and exists when the components are homologues, e.g. polyethylene, and, say, n-pentane, or polystyrene and toluene. The free volume difference is due to the difference in structure between the polyethylene and the pentane. Suppose the pentane molecules were to be connected by valence forces to form polyethylene molecules in the liquid state. During the process, external, volume-dependent degrees of freedom of the pentane molecules are replaced by low-amplitude vibrations internal to the polyethylene chain. These do not affect the volume: hence there is a decrease of the thermal energy promoting expansion of the liquid. However, there is a small counter-eflect. The disappearance of the ends of the pentane molecules reduces the total molecular surface available for intermolecular contacts.

This effect would decrease the total cohesive energy resisting the thermal expansion. Both effects can be taken into account through the ratio of the number of external degrees of freedom, $3 c$, of the molecule, to the number of external contacts, $q z$. The ratio is, within a constant factor, the Prigogine structural parameter, $c / q$. This parameter decreases in passing from a monomeric liquid to a polymeric one.

One may also consider the ratio of the thermal energy of the external degrees oflireedom to the cohesive energy. This gives the reduced temperature :

$$
\tilde{\Gamma}=\frac{U_{\text {external }}}{U_{\text {cohesive }}}=\frac{c}{q} \frac{k T}{\varepsilon^{*}}=\frac{T}{T^{*}}
$$

Here $:^{*}$ is the depth of the interaction potential between segments or essentially a characteristic cohesive energy per contact. The reduced temperature is directly related through the equation of state to the reduced volume, $\tilde{l}=V^{*} V^{*}$, where the molar reduction parameter, $V^{*}$, may be thought of as the actual molecular volume. One thus sees that $\tilde{T}$ of the liquid is characteristic of its free volume or degree of thermal expansion. The free volume of a series of homologues will decrease with increasing chain-length, not through any change of the force-lield around the homologues, i.c. $\varepsilon^{*}$ in equation 1. but through the decrease of the structural parameter, $r / q$. Furthermore. the decrease depends on the llexibility of the chain, that is, on the extent to which the valence bonds reduced the number of external degrees of freedom. Molecular structure and size alfect the properties of the liquid by changing c/q. On the other hand, two 'monomeric' liquids, e.g. ethane and ethanol. are of widely different free volume, not because of a large difference in $c / q$, but because of a difference in intermolecular force fields or $\varepsilon^{*}$.

The dilference of free volume, whether it arises from a difference of $c / q$ or of $\varepsilon^{*}$. has an important role in determining the thermodynamic mixing functions of the solution. During the mixing process, the free volumes of the two components approach an intermediate value characteristic of the mixture. These changes of free volume lead not only to an over-all change of volume. $\Delta V_{M}$, but also to important contributions in the other mixing functions, $\Delta H_{M}, \Delta S_{M}$ and $\Delta G_{M}$. The main features of the contemporary view of the thermodynamics of non-dilute polymer solutions were set forth filteen years ago in Prigogine's book: 
The origin of the excess properties has to be found in the following three effects :

Geometrical effect: the molecular volumes of A and B are different; hence the combinatorial factor is different from that of an ideal system. This is a purcly geometrical effect present in all $r$-mer systems and independent of the natures of $\mathrm{A}$ and $\mathrm{B}$...

Energetical effect: the interactions between pairs of elements AA, AB and $B B$ are different; this kind of effect is exactly the same as in monomer mixtures...

Structural effects: the ratios $c_{\mathrm{A}} / q_{\mathrm{A}}$ and $c_{\mathrm{B}} / q_{\mathrm{B}}$ may be different; in this case, we also have deviations from ideality. This effect. like the geometrical effect. is specific to $r$-mer mixtures. However, the geometrical effect is general and independent of the nature of A and B, while the structural effect is directly related to the structure of the molecules and depends primarily on the nature of the valency forces.

It is of interest to note that both characteristically polymer effects. the geometrical and the structural, raise the free energy of the mixture above a corresponding monomeric system with the same quantity of material, that is, the free energy is increased by linking particles of component 2 to form chains (where 1 and 2 denote the solvent and polymer).

We should appreciate that the energetic effect can be divided into two parts. (a) One would be an eflect of the relative weakness of 1-2 contacts compared with $1-1$ and $2-2$ contacts or $\varepsilon_{12}^{*}<\frac{1}{2}\left(\varepsilon_{11}^{*}+\varepsilon_{22}^{*}\right)$, corresponding to a non-zro value of the 0 -parameter in the Prigogine nomenclature or the $X_{12}$-parameter in the Flory nomenclature. This is the effect which gives rise to the interchange energy, $\Delta w$ in the traditional Flory-Huggins theory or the theory of strictly regular solutions. (b) However, even if $\varepsilon_{11}^{*}=\frac{1}{2}\left(\varepsilon_{11}^{*}\right.$ $\left.+\varepsilon_{22}^{*}\right)$ there is still an energetic effect if $\varepsilon_{11}^{*} \neq \varepsilon_{22}^{*} ;$ the $\widetilde{T}$ and free volumes of the liquids are different. This energetic effect and the structural effect act similarly through the free volume. Although it is conceptually important to separate the roles of $\varepsilon^{*}$ and $c / q$ in $\tilde{T}$, it is difficult to do so operationally. It is thus useful to distinguish only the following effects on the mixing functions:

(1) the combinatorial or geometrical effect.

(2) the effect of an energetic weakness of contacts of unlike type.

(3) effect of free volume difference between the components due to a difference of $c / q$ or $\varepsilon^{*}$ in $\tilde{T}$.

Contributions (2) and (3) are now similar in form for monomeric and polymeric solutions. They give the well-known $\chi$-parameter which the Flory-Huggins theory attributed exclusively to contribution (2). Their theoretical treatment has been given by Prigogine and Flory, and more specifically for monomeric mixtures by $\mathrm{Scott}^{4}$ and Brown ${ }^{5}$.

\section{LOWER CRITICAL SOLUTION TEMPERATURES}

Prigogine and his colleagues were primarily interested in qualitative predictions and interpretations of new phenomena. One of these is the lower critical solution temperature (LCST) which occurs as a general phenomenon in polymer solutions in addition to the better known upper critical solution temperature (UCST) associated with the Flory $\Theta$-point. The UCST is related to a positive $\Delta H_{M}$ arising from contribution (2), i.e. a weakness of 


\section{PATTERSSON}

unlike contacts. The LCST is related ${ }^{\mathrm{t}}$ to a negative contribution to the $\Delta S_{M}$. and is associated with contribution (3). The necessary difference of free volume or $\tilde{T}$ may be at tained at higher temperatures due to the difference in structural parameters between the components. This would be the case in polymer solutions. LCST are also found ${ }^{7.8}$ in mixtures of higher branched and normal alkanes with methane. ethane and propane and here again the free volume difference is presumably a structural effect $\dagger$. On the other hand. the LCST was first studied in ethane-ethanol ${ }^{12}$ mixtures. Here the free volume difference is due to the difference of contact energies. $\varepsilon^{*}$, in the $\tilde{T}$ expression.

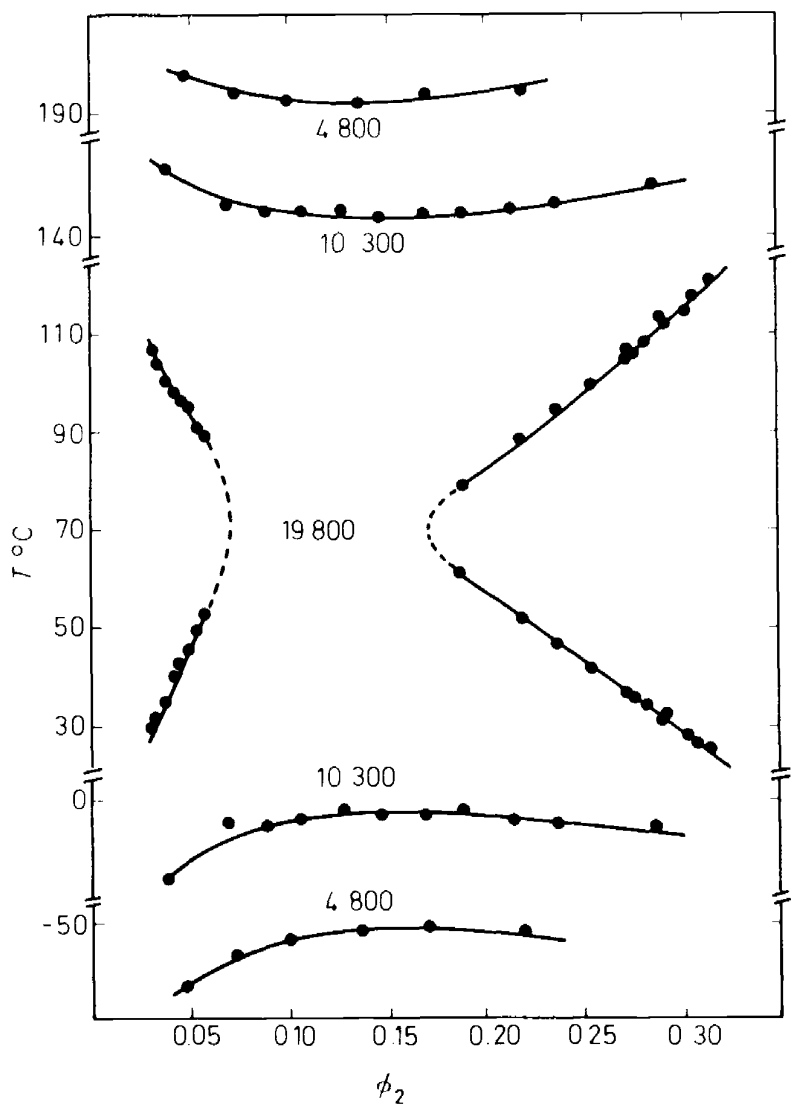

Figure 1 . The (temperature. volume fraction) phase diagram for the polystyrene acetone system. for fractions of indicated MW. showing UCST and LCST for low MWs and the hour-

glass" cloud-point curve for the 19800 fraction. The LC $\mathrm{ST}$ are the higher temperatures.

+ Confusion can arise from the term "energy'. Thus Rowlinson" suggests that the LCST in hydrocarbon mixtures is primarily an energetic eflect. Here the energy is that of the whole chainmolecule. i.c. $\varepsilon^{*}$ times the number of segments. The energy per segment is essentially the same for the two components. The large difference in energies between methane and e.g. hexane is due to the large difference in numbers of segments. (See also discussion in refs. 10 and 11 ). 
Figure $I$ shows the phase diagram ${ }^{13}$ for several fractions of polystyrene in a poor solvent, acetone. Diethyl ether gives a similar phase diagram ${ }^{13}$. These solvents are poor, in part because they are of different chemical nature from the polystyrene. However, a more important factor is their large degree of thermal expansion as indicated by large values of the thermal expansion coeflicient. (At $25^{\circ} \mathrm{C}, \alpha=1.42 \times 10^{-3}$ and $1.67 \times 10^{-3} \mathrm{~K}^{-1}$ for respectively, acetone and diethyl ether.) Thus, both a UCST and an LCST are found, rellecting a minimum in the $\chi$-parameter as a function of temperature and attainment of the critical value of $\chi$ at two temperatures. With the Flory-Huggins assessment of the combinatorial contribution to $\Delta G_{M}$ we have

$$
\chi_{\mathrm{crit}}(P, T)=\frac{1}{2}\left(1+r^{-\frac{1}{2}}\right)^{2} .
$$

As the molecular weight of the polymer is increased, the combinatorial contribution to the free energy per mole of segments increases, and hence solution stability decreases, corresponding to a decrease of the critical value of $\chi$ in equation 2. In Figure 1, the UCST and LCST move together until at a molecular weight just below 19800 the two critical.solution temperatures coalesce. The two-phase region then has an 'hour-glass' shape, and there is no temperature region of complete polymer-solvent miscibility. With further increase of the molecular weight the right-hand side of the hour-glass moves rapidly to higher concentration and the left-hand side to such low concentration that it is inconvenient to measure it. This type of phase diagram should be general for a polymer in a non-solvent.

The polymer will swell to a concentration given by the right-hand side of the hour-glass, and on increasing the temperature will at lirst imbibe more solvent and then shrink. These phenomena are also obtained with crosslinked networks.

The Flory-Shultz plot ${ }^{14}$ of $T_{c}^{-1}$ against $r^{-\frac{1}{2}}+(2 r)^{-1}$, where $r$ is the number of segments of the polymer, is expected to be a straight line of slope $\left(\psi_{1} \theta\right)^{-1}$. Here the $\psi_{1}$-parameter is proportional to the entropy of dilution and is hence positive at the UCST but negative at the LCST. Figure 2 shows $T^{-1}$ for the systems, polystyrene-acetone and polystyrene-diethyl ether and for polystyrene-dimethoxymethane which shows the LCST but no UCST above $-78^{\circ} \mathrm{C}$. One notices that in the first two systems the $\psi_{1}$-parameter has a marked temperature dependence, decreasing as the temperature is raised through the UCSTs and then becoming negative for the LCSTs. Thus the form:

$$
\left(\frac{1}{2}-\chi_{1}\right)=\psi_{1}\left(1-\theta_{/} I\right),
$$

also used in applying excluded volume theories, is not of general validity.

Diagrams similar to those in Figure 1 can be obtained with a single molecular weight of polymer, but adding a solvent of lower thermal expansion to form a ternary system. Wolf ${ }^{15}$ has illustrated this with the polystyrene -acetone-methyl ethyl ketone system. The methyl ethyl ketone $(\alpha=1.29$ $\times 10^{-3} K^{-1}$ at $25 \mathrm{C}$ ) having a lower degree of thermal expansion than the acet one, lowers the free volume contribution to $\chi$ throughout the temperature range. The LCST is raised and the UCST lowered, so that qualitatively one has the same effect as obtained by decreasing the molecular weight of the polymer. 


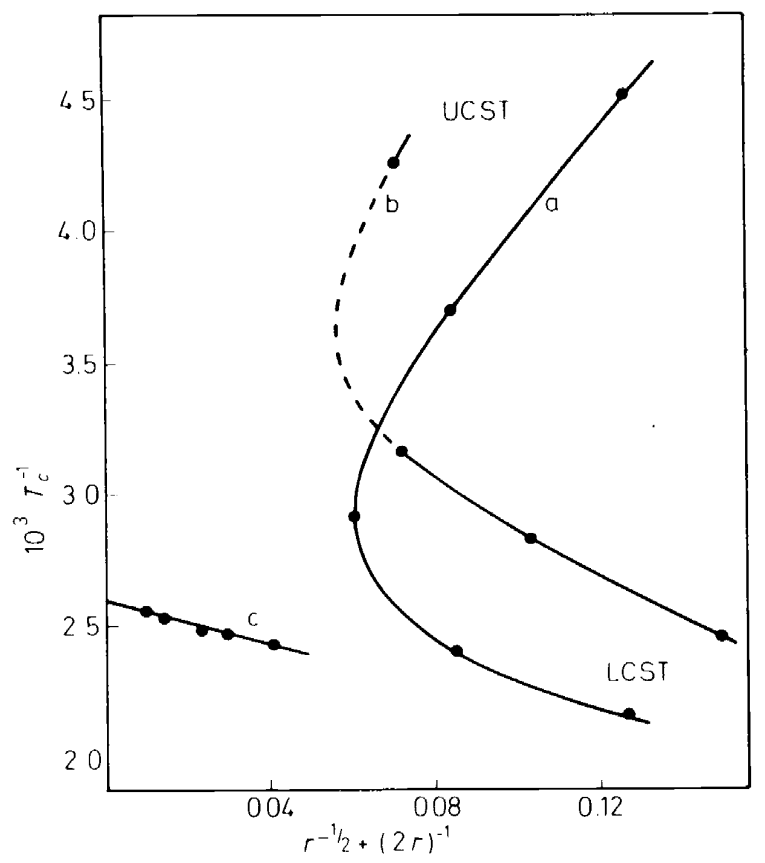

Figure 2. The Shultz Flory plot of reciprocal critical solution temperature against $r+12 r) 1$ for: a, polystyrene acetone: b. polystyrene diethyl ether: and c. polystyrene dimethoxymethane. The dotted line represents an estimated section of the curve.

\section{PRESSURE EFFECTS}

\section{Effect of pressure on phase diagrams}

The pressure dependence of the LCST has been published for a number of systems: polyethylene-pentane ${ }^{16}$, polyisobutylene-2-methylbutane ${ }^{17}$ and polystyrene-methyl acetate ${ }^{18}$. Since the polymer is less compressible than the solvent, application of pressure decreases the free volume difference between the components, and therefore displaces the LCST to higher temperature. Ehrlich ${ }^{16}$ has pioneered in investigating the solubility of polyethylene in solvents which are gases under normal pressure and temperature: ethane, propane, butane and ethylene. With pressure, the free volume difference between polymer and solvent is decreased sufficiently to allow the polymer to enter the solution. Striking effects are found when different polymers are used. For instance, ethane ${ }^{16}$ at $100^{\prime} \mathrm{C}$ requires a pressure of some $1200 \mathrm{~atm}$ to solubilize polyethylene of high molecular weight. However, only $100 \mathrm{~atm}$ are required for polydimethylsiloxane ${ }^{19}$ which has a higher free volume than polyethylene, as indicated by its higher thermal expansion coefficient $\left(\alpha=9 \times 10^{-4} K^{-1}\right.$ for PDMS at $25^{\circ} \mathrm{C}$ while an extrapolated value for liquid polyethylene would be $7 \times 10^{-4} K^{-1}$ ). Ehrlich has pointed out that the LCST at the saturation pressure of the solvent actually lies on 
a critical line - a continuous line of critical solution temperatures which passes through a maximum in pressure and would linally arrive at the critical point of the pure polymeric liquid. This point would be unattainable in the case of a high polymer, due to degradation. The phase diagrams of polymer solutions are not different in character from those of small-molecule mixtures which have been studied extensively in chemical engineering ${ }^{7}$. In fact theories ${ }^{8.20}$ of the phase relations need make no real distinction between the two types of solutions.

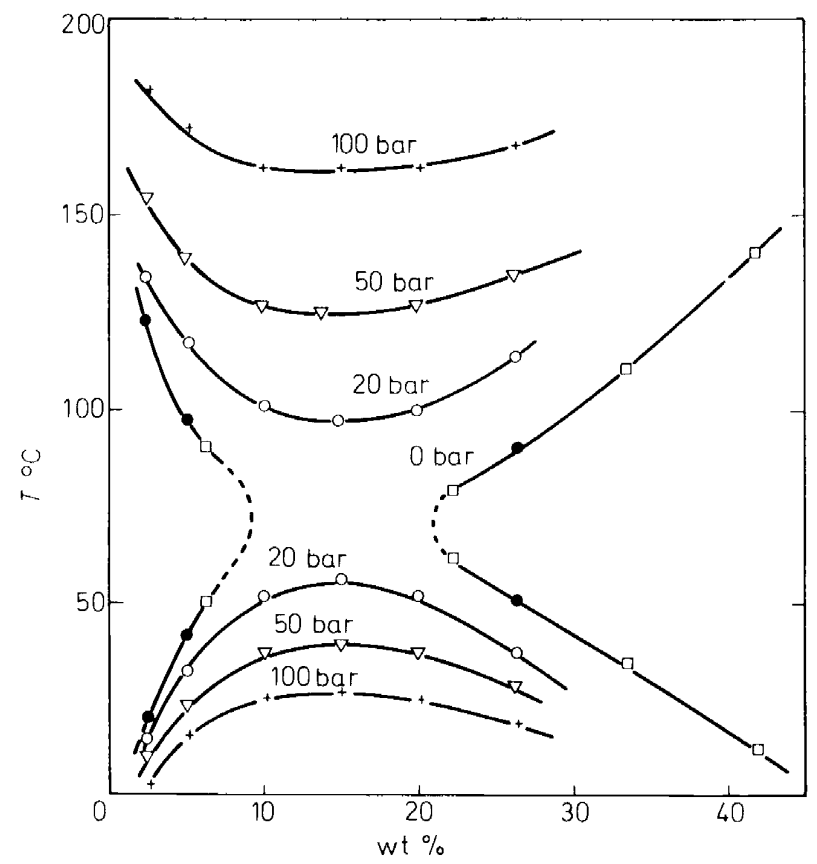

Figure 3. (Temperature, weight $\%$ ) cloud-point curves for polystyrene of MW 20400:- 0 bar; $\checkmark-20$ bar; $\nabla-50$ bar; +-100 bar; $\square$ - results from ref. 13 extrapolated from the solvent saturated vapour pressure to 0 bar.

Pressure has a very large effect ${ }^{19}$ on the polystyrene-acetone phase diagram in Figure 1. In Figure 3 the same hour-glass two-phase region is seen at zero pressure (actually in these experiments with a $20400 \mathrm{MW}$ fraction). On applying only 20 bar, the polymer-solvent compatibility is increased so that the UCST and LCST are separated by a $40^{\circ} \mathrm{C}$ gap. Higher pressures increase the gap and the cloud-point curves take up the same positions as were obtained in Figure 1 with fractions of lower molecular weight or through adding methyl ethyl ketone to the acetone. By applying pressure or adding a less expanded solvent the $\chi$-parameter in equation 2 is decreased, while by decreasing the molecular weight the critical value of $\chi$ is increased. 


\section{PATTERSON}

Eflects of pressure on the phase diagrams of non-polymeric mixtures have been extensively studied by G. M. Schneider ${ }^{21}$. In particular the hourglass cloud-point curves are general features in systems where there is a free volume difference and also a disparity of intermolecular forces between the components. Thus, Figure 3 for polystyrene-acetone is very similar to Figure 6 of ref. 22 for the methylcyclopentane-methane system.

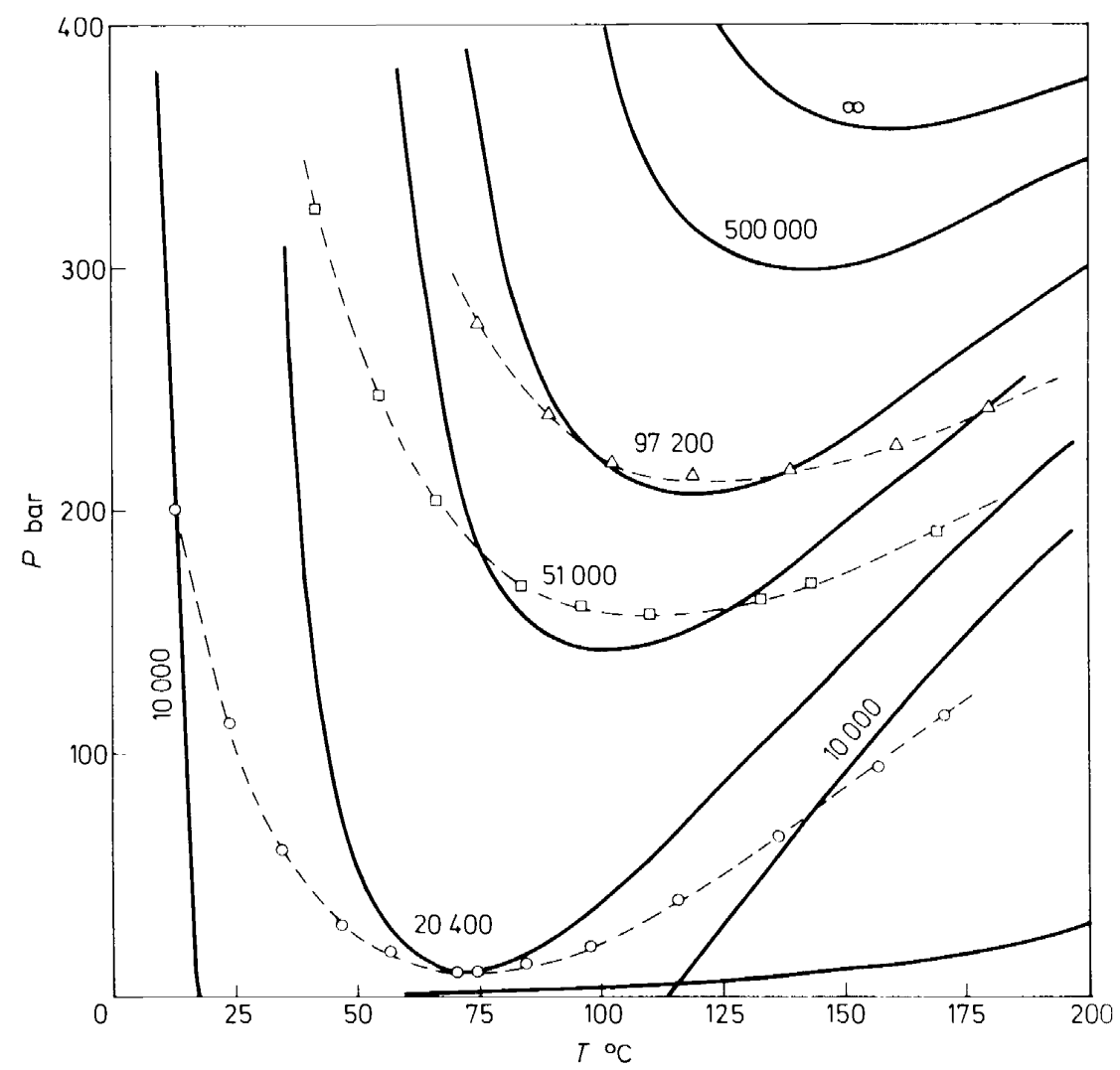

Figure 4. $(P . T)$ for different fractions of polystyrene in acetone. Experimental points: ${ }^{\circ} \quad \mathrm{M}$ iN

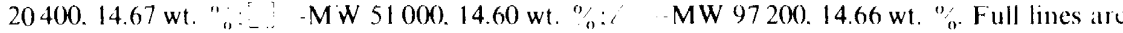
calculated from the theory with $X_{12}=4.483$. Thin line: Saturated vapour pressure of acetone.

The effect of pressure with different molecular weights of polystyrene in acetone may best be illustrated by experiments at a single composition to determine the pressure which allows the polymer and solvent to mix. In Figure 3, the maxima and minima of the cloud-point curves occur at $\sim 14 \%$ by weight. Figure 4 shows the $P(T)$ results at this concentration for polymer of molecular weights 20400,51000 and 97200 . On the $P(T)$ curve for each molecular weight, points to the left of the minimum correspond to UCST 
and $\Delta H_{M}>0, \overline{\Delta S_{1}}>0$, while those on the right correspond to LCST, where $\Delta H_{M}<0$ and $\overline{\Delta S_{1}}<0$. Throughout the temperature range of Figure $4, \Delta V_{M}<0$, since the polymer-solvent compatibility is increased by pressure. With increase of the molecular weight of the polymer fraction, the $P(T)$ curves are moved to higher $P$ and $T$. The increase of $P$ is of course a consequence of the decrease in the critical value of $\chi$ as given by equation 2 . The shift to higher $T$ is associated with the change in the relative importance of the contributions to $\chi$ from the weakness of the 1-2 contacts and from the free volume difference. At higher pressure the first contribution increases, and the second decreases in importance. Hence there is a displacement of the curve to higher temperature. With systems where the disparity in intermolecular forces is greater than in polystyrene-acetone, one would expect to reach a $P(T)$ curve where $(\mathrm{d} P / \mathrm{d} T)_{c}$ is negative throughout and the region of LCSTs would have disappeared. The $P(T)$ curves for polystyrene fractions of increasing molecular weight in acetone are analogous to $P(T)$ curves for hydrocarbons of increasing chain-length in methane, e.g. 1-hexene, 1-heptene ${ }^{22}$. or increasing disparity of force fields, e.g. 1-hexene, methylcyclopentane, toluene (cf. Figure 16 of ref. 21).

\section{Effect of pressure on the second virial coefficient and chain dimensions}

It is clear from the phase diagrams that the thermodynamic mixing functions of polymer solutions should be strongly dependent on pressure as well as temperature. The light scattering technique is suitable for studying this effect. Schulz and Lechner ${ }^{23}$ have pioneered in measuring the second virial coefficient, $A_{2}$, and the radius of gyration, $\left\langle S^{2}\right\rangle^{\frac{1}{2}}$ of macromolecules at pressures up to $800 \mathrm{~atm}$. Claessen and collaborators have reached much higher pressures, and their measurements should provide stringent tests of polymer solution theories which endeavour to account for free volume

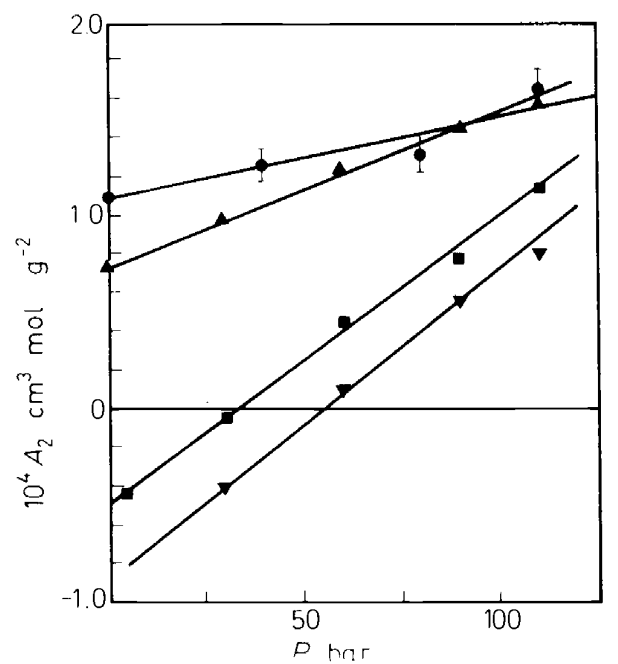

Figure 5. Values of $A_{2}$ as a function of pressure for: 9 , polystyrene $(975000 \mathrm{MW})$ in methyl ethyl ketone at $22 \mathrm{C}^{\circ} \mathbf{\Delta}$. polyisobutylene $(1010000 \mathrm{MW})$ in 2-methylbutane at $24 \mathrm{C}: \mathbf{\square}$. at $57^{\circ} \mathrm{C}: \nabla$, at $64^{\circ} \mathrm{C}$. 
effects. Results of some thermodynamic interest may be obtained using conventional light scattering apparatus and glass cells capable of withstanding $-150 \mathrm{~atm}$. The usual Zimm-plot technique gives the value of $A_{2}$ and $\left\langle S^{2}\right\rangle^{\frac{1}{2}}$. For the PIB-2-methylpentane system at saturation vapour pressure ${ }^{2+} . A_{2}$ (shown in Figure 5 ) and $\left\langle S^{2}\right\rangle^{\frac{1}{2}}$ decrease with increase of temperature. $A$ zero value of $A_{2}$ is attained at about $44 \mathrm{C}$ which is thus a $\Theta$-point associated with the LCST. If pressure is applied. both $A_{2}$ and $\left\langle S^{2}\right\rangle^{\frac{1}{1}}$ are markedly increased. The temperature dependence of $A_{2}$ is related to the heat of dilution or the relative partial molar heat of the solvent. $\bar{\Delta} \bar{H}_{1}$. and hence to $(\hat{\imath} \chi T)_{p}$. In the same way. the pressure dependence of $A_{2}$ is related to the relative partial molar volume of the solvent. $\Delta V_{1}$ and hence

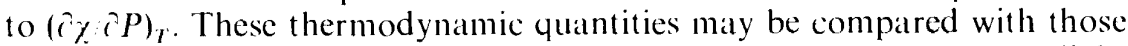
obtained using calorimetric and dilatometric methods. However, light scattering measurements are usually made at very low concentration. It therefore seems that the solution heterogeneity should be taken into account through the $h(z)$ function of one of the dilute solution theories ${ }^{25}$. When this is done. fair agreement is obtained ${ }^{24}$.

The correlation of the change of $\left\langle S^{2}\right\rangle^{\frac{1}{2}}$ with the change of $A_{2}$ is also of interest since these quantities are related through the excluded volume parameter. $z$ or in fact $\%$. If neither pressure nor temperature has an effect on the unperturbed dimensions of the coil. a single curve for all systems should be obtained for the coil expansion coefficient. $x$ against $A_{2} A^{-3} M^{\frac{1}{2}}$. where $A$ is related to the unperturbed end-to-end distance through ${ }^{25}$ $A \equiv\left(\left\langle L_{0}^{2}\right\rangle M\right)^{\frac{1}{3}}$. A single curve seems to be obtained in our work with PIB 2-methylbutane and polystyrene methyl ethyl ketone. On the other hand. for polystyrene-toluene and polystyrene trans-decalin. Schuly and Lechner ${ }^{2.3}$ find a definite decrease of $\left\langle S_{0}^{2}\right\rangle^{\frac{1}{2}}$ with increase of pressure. This seems to imply an effect of the solvent molecule packing on the unperturbed dimensions of the polymer. It is generally believed that the solvent has very little effect on the equilibrium between the different rotational isomers in the chain. but the possibility cannot be dismissed ${ }^{26}$. An eflect of solvent packing on this equilibrium would have important consequences in polymer solution thermodynamies, and would give a new role to the free volume.

\section{QUANTITATIVE ASPECTS OF POLYMER SOLUTION THERMODYNAMICS}

\section{Combinatorial contribution}

The most important contribution to the entropy and free energy of mixing is the 'geometrical effect' mentioned by Prigogine. i.e. the combinatorial contribution in the lattice terminology. It is usual to compute this contribution through the Flory Huggins expression:

$$
\Delta G_{M}(\mathrm{comb})=R T\left(n_{1} \ln \phi_{1}+n_{2} \ln \phi_{2}\right)=-T \Delta S_{M}(\mathrm{comb})
$$

Combining this with a non-combinatorial term one has the well-known expression for the chemical potential of the solvent:

$$
\begin{aligned}
\Delta \mu_{1} R T=\ln \phi_{1}+\left(1-r^{1}\right) \phi_{2} & +\chi \phi_{2}^{2} \\
(\text { comb) } & +(\text { non-comb) }
\end{aligned}
$$


Initially, the $\phi$ were taken as volume fractions of the components. These variables are. strictly speaking, temperature and pressure dependent at constant mole or weight fraction. This follows from the thermal expansion coefficient and compressibility of the polymer being much less than for the solvent. However, in calculating $\Delta S_{M}(\mathrm{comb})$ within the context of the lattice model, the volume fractions were replaced by fractions ${ }^{27}$ in molecular volumes or numbers of molecular segments. These fractions are independent of $T$ and $P$. and this procedure has been taken over in applying ${ }^{28.2}$ the Prigogine and recent Flory theories. The actual volumes of the components are replaced by the volume reduction parameters $V^{*}$ (core volumes) giving the 'segment fractions' in the Flory terminology, i.e.

$$
\phi_{1}=n_{1} V_{1}^{*} /\left(n_{1} V_{1}^{*}+n_{2} V_{2}^{*}\right)
$$

instead of

$$
\phi_{1}=n_{1} v_{1}^{*} \tilde{V}_{1} /\left(n_{1} V_{1}^{*} \tilde{V}_{1}+n_{2} V_{2}^{*} \tilde{V}_{2}\right)
$$

Using the two prescriptions for $\phi$ leads to values of the $\chi$ parameter, calculated from experimental $\Delta \mu_{1}$ data, which can differ by as much as 0.1 to 0.2 . and the concentration dependences of $\chi$ are slightly different. $A$ difference of free volume between the components is meaningless within the lattice model on which equations 3 and 4 were based, and it does not seem obvious which of the two prescriptions is correct. The "lollowing two arguments lavour the segment fractions.

First, the $T$ and $P$ dependence of the volume fractions has peculiar consequences. There would be combinatorial contributions ${ }^{29.30}$ to $\Delta H_{M}$ and $\Delta V_{M}$ arising from $\Delta G_{M}$ (comb). They would be negative ${ }^{29}$ and of the same magnitude as experimentally observed mixing functions. Also, the FloryHuggins expressions in equation 3 for $\Delta G_{M}$ and $\Delta S_{M}$ would be inconsistent since $\left(\partial \Delta G_{M} / \partial T\right)_{P}$ would not give $-\Delta S_{M}$.

$\Lambda$ stronger argument in lavour of the segment fractions comes from considering the Longuet-Higgins derivation ${ }^{31}$ of equation 3. This derivation is well known since it does not assume the lattice model. However, the most important and unrealistic leature of the model remains. The two components and the mixture must all have the same 'density', i.e. the same degree of thermal expansion or free volume. Thus there is still no distinction between volume and segment fractions. However, the derivation can still be used to give the $\Delta S_{M}$ in the real case of components of unequal free volume, and it appears that the segment fraction is the appropriate composition variable. The mixing of components of different free volume could take place in a three step process, which is the second of the constant-volume processes considered by Scott ${ }^{4}$ and McGlashan ${ }^{32}$. termed constant volume per element (segment) by McGlashan. Step 1 consists of compression or expansion of the components to the same standard free volume: step 2. mixing at equal free volume; step 3. expansion or compression of the mixture to its equilibrium free volume. The value of $\Delta S_{M}$ associated with step 2 is, according to Longuet-Higgins, given by the Flory-Huggins equation. The $\phi$ to be used are numerically equal to the segment fractions in the original components of different free volume. The entropy effects 


\section{PATTERSON}

associated with steps 1 and 3 are exactly the free volume effects, which are to be dealt with in a supplementary term.

It is interesting to consider the $\Delta S_{M}$ in a case of ext remely large free volume: the components are now two dilute gases at equal pressure, $P_{0}$, one having monomeric molecules and the other $r$-mers. It is clear, intuitively. that $\Delta S_{M}$ must be ideal, but it is not obvious how this is to be arrived at from the Flory-Huggins equation. Equal pressure means equal numbers of molecules/volume whereas in the Longuet-Higgins derivations equal density has the significance of equal free volume or equal numbers of segments/volume. At equal $P_{0}$, the number of segments/volume for the polymeric gas is $r$ times higher than for the monomeric gas. Step 1 therefore expands the volume of the $n_{2}$ moles of $r$-mers by a factor of $r$ :

$$
\Delta S / R=n_{2} \ln r .
$$

Using equation 3, step 2 gives $\Delta S R=-n_{1} \ln n_{1}\left(n_{1}+r n_{2}\right)-n_{2} \ln r n_{2}$ $\left(n_{1}+r n_{2}\right)$. Step 3 must compress the mixture by a factor of $\left(n_{2} r+n_{1}\right)$ $\left(n_{1}+n_{2}\right)$ to regain the original pressure $P_{0}$ :

$$
\Delta S / R=-\left(n_{1}+n_{2}\right) \ln \left(n_{1}+n_{2} r\right) /\left(n_{1}+n_{2}\right) .
$$

The sum of the entropy changes is :

$$
\Delta S / R=-n_{1} \ln x_{1}-n_{2} \ln x_{2} .
$$

As predictions of the $\chi$-parameter become accurate, it will be important to consider more exact approximations for the combinatorial $\Delta S_{M}$, due to Huggins. Miller, Orr. Guggenheim (presented in the texts by Guggenheim ${ }^{27}$ and Tompa ${ }^{33}$ ) and Kurata ${ }^{34}$. In computing the $\chi$-parameter values from solvent activity data. it is of importance to assess the effect on the combinatorial entropy of branching of the polymer or solvent, differences of chain-diameter and size between the components, and differences of flexibility. Some of these effects are discussed in the texts by Guggenheim and Tompa. The exact calculations, using a 2-dimensional square lattice, of Bellemans and co-workers ${ }^{35}$ should lead to further insights.

\section{Non-combinatorial contributions at zero pressure}

In polymer solution work, the partial molar mixing quantitics are more useful than the actual mixing quantities. The non-combinatorial contributions to $\Delta \mu_{1}, \Delta H_{1}$ and $-T \Delta \bar{S}_{1}$ define interaction parameters:

$$
\begin{aligned}
\left(\Delta \mu_{1}\right)_{\text {non-comb }} & \equiv R T \gamma_{\phi}^{2} \\
\left(\Delta \bar{H}_{1}\right)_{\text {non-comb }} & \equiv R T \chi_{H} \phi_{2}^{2} \equiv R T \kappa \phi_{2}^{2} \\
-T\left(\Delta \bar{S}_{1}\right)_{\text {non-comb }} & \equiv R T \chi_{s} \phi_{2}^{2} \equiv R T\left(\frac{1}{2}-\psi\right) \phi_{2}^{2}
\end{aligned}
$$

Thus.

$$
\chi=\chi_{. H}+\chi_{s}=\kappa+\frac{1}{2}-\psi
$$

The $\kappa$ and $\psi$ are from the nomenclature used by Flory, who has also recently used $^{2 a}$ the $\chi_{H}$ and $\chi_{s}$ nomenclature. The latter originates, we believe, with Tompa $^{3.3}$.

The corresponding-states approach of Prigogine gives similar expressions 
of identical form for the three partial molar quantities (equation 23 of ref. 36):

$$
\begin{aligned}
& \left(\Delta \mu_{1}\right)_{\text {non-comb }} \equiv R T \chi \phi_{2}^{2}=P_{1}^{*} V_{1}^{*} \mid\left\{\tilde{G}(\tilde{T})-\tilde{G}\left(\tilde{T}_{1}\right)+\left(\tilde{T}_{1}-\tilde{T}\right)(\partial \tilde{G} / \partial \tilde{T})_{\tilde{P}}\right\} \\
& +\left\{-\tilde{G}(\widetilde{T})+\tilde{T}(\partial \widetilde{G} / \partial \widetilde{T})_{P}\right\}\left(\frac{X_{1}^{2}}{P_{1}^{*}} \theta_{2}^{2}\right) \mid .
\end{aligned}
$$

The expressions for $\Delta \bar{H}_{1}$ and $T \Delta \bar{S}_{1}$ are obtained by replacing the reduced function $\widetilde{G}$ by $\widetilde{H}$ and $\widetilde{T S}$, respectively. Equation 8 has been written with the nomenclature of the more recent Flory theory. In the Prigogine nomenclature, $\theta_{2} \equiv X_{2}$ : both correspond to the surface fraction of polymer, i.e. the fraction of the molecular surface associated with the polymer. Prigogine used a lattice model to calculate this quantity while the Flory approach considers the actual molecular structures. Previously, we had ${ }^{36}$

$$
P_{1}^{*} V_{1}^{*} \equiv U_{1}^{*}, X_{12} / P_{1}^{*} \equiv v^{2} .
$$

The first term in equation 8 corresponds to the equation of state term of Flory and is associated with the free volume changes : the second corresponds to the contact interaction term of Flory, associated with a weakness of the 1-2 contacts. The expressions may be written in terms of a particular model of the liquid state, e.g. that of Flory which puts:

and

$$
\begin{gathered}
\tilde{U}=-\tilde{V}^{-1} \\
\tilde{S}=3 \ln \left(\tilde{V} \frac{1}{3}-1\right) .
\end{gathered}
$$

The corresponding expressions of the Flory theory. i.e. equations 46-48 of ref. $2 \mathrm{~b}$ are then obtained from equation 8 . They give a very good semiquantitative interpretation of the partial molar quantities as seen in the work of Flory and collaborators. However, it may still be advantageous to have the more general equations in order to assess discrepancies between theory and experiment. They permit a visualization of the thermodynamic origin of the $\chi_{,} \chi_{H}$ and $\chi_{S}$ parameters as illustrated in Figure 6 for the calculation of $\chi$. It may be seen that the first term of equation 8 has the opposite sign to the curvature of $\widetilde{G}(\widetilde{T})$ against $\widetilde{T}$, i.e. it has the sign of $\widetilde{C}_{p}$, the reduced configurational heat capacity, which is a positive quantity. The contact interaction term must also be positive since

$$
-\tilde{G}(\tilde{T})+\tilde{T} \partial \tilde{C}_{p} / \partial \tilde{T}=-\tilde{U}
$$

and the configurational energy, $U$, of any liquid is negative. The signs of the two terms in the $\chi_{,} \chi_{H}$ and $\gamma_{s}$-parameters are noted in the following table.

Table 1. Signs of the terms in the $\chi_{.} \chi_{H}$ and $\chi_{s}$-parameters

\begin{tabular}{lccc}
\hline & $\chi$ & $\chi_{H}$ & $\chi_{S}$ \\
$\begin{array}{l}\text { Equation of state } \\
\text { term proportional to }\end{array}$ & $\tilde{C}_{p}$ & $\begin{array}{c}-\tilde{T} \partial \tilde{C}_{p} / \partial \tilde{T} \\
(- \text { at high } \tilde{T})\end{array}$ & $\begin{array}{c}\tilde{T} \partial C_{p} / \partial \tilde{T}+\tilde{C}_{p} \\
(+ \text { at high } \tilde{T})\end{array}$ \\
$\begin{array}{l}\text { Contact } \\
\text { interaction } \\
\text { term proportional to }\end{array}$ & $-\tilde{U}$ & $-\tilde{U}+\tilde{T} \tilde{C}_{p}$ & $-\tilde{T} \tilde{C}_{p}$ \\
\end{tabular}




\section{PATTERSON}

In $\chi_{s}$, the contact interaction term. important in monomeric systems, is negative. while the equation of state term must be positive at higher $\widetilde{T}$ since $\mathrm{d} \widetilde{C}_{p} / \mathrm{d} \widetilde{T}$ tends to infinity at the vapour liquid critical point of the liquid. In the Flory model, $\partial \widetilde{C}_{p} / \partial \widetilde{T}$ and hence $\chi_{s}$, are positive for all $\widetilde{T}$. For a typical polymer solution the major part of $\chi$ is contributed by the entropic $\chi_{s}$.

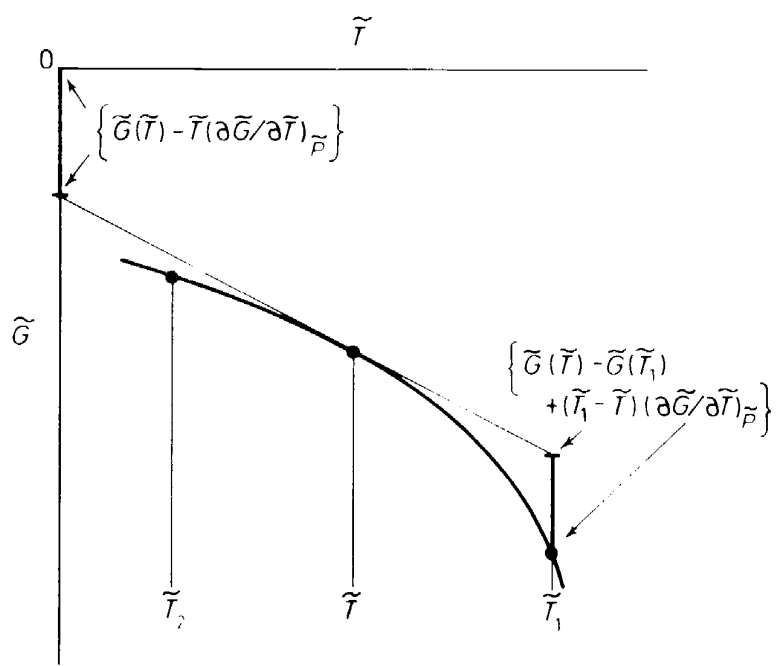

Figure 6 . Visualization of the terms in $\left(\Delta \mu_{1}\right)_{\text {non-comb }}$ in equation 8 . The length $\tilde{G}(\tilde{T})-\tilde{T}(\hat{G} \tilde{G}, \tilde{T})_{p}$ enters equation 8 with positive sign and is multiplied by the dimensionless parameter $\chi_{12} P_{1}^{*}$ to give the contact interaction term.

Using calorimetry and the vapour sorption technique ${ }^{37}$, we have measured heats of dilution and solvent activities for about a dozen systems composed of polydimethylsiloxane with the aliphatic and aromatic hydrocarbons and and siloxane oligomers. Quite generally, the $\chi$ values are much larger than would be predicted by the Flory model, or any other (a variety of models give very similar predictions). The thermal expansion coefficient of the polymer is so large that it is more typical of a solvent than a polymer. The free volume contribution is therefore very small in the predicted $\gamma$. In many of the systems the $\chi_{s}$ parameter is predicted to be negative. The experimental values obtained at $25 \mathrm{C}$ for $\chi$ and $\chi_{s}$ are, however, large and typical of polymer-solvent systems. Furthermore. Morimoto ${ }^{38}$ has obtained volumes of mixing of PDMS with some ten solvents at high dilution of the polymer. In all cases but one the volume of mixing is negative, whereas the theory, independent of the model, predicts positive volumes of mixing.

We have also obtained heats of dilution ${ }^{30}$ of polyisobutylene and polystyrene in a number of solvents. Several of these solutions have negative heats of dilution, e.g. PIB $n$-pentane, PDMS-n-pentane, PS toluene. In all of the solutions the $\kappa$ or $\chi_{H}$ is predicted to increase with increasing polymer 
concentration. This is strikingly so in the case of the PIB-n-pentane where $\chi_{H}$ is predicted ${ }^{2 e}$ to vary between -0.4 and +0.2 . (The average value -0.1 is fitted to the experimental heat of solution.) According to our measurements, however, the $\chi_{H}$ is almost independent of concentration, remaining throughout equal to -0.1 . In the PS-toluene case, $\chi_{H}$, already negative for $\phi_{2}=0$, becomes more negative with increasing polymer concentration ${ }^{39.36}$. In thinking qualitatively about the concentration dependence of a negative $\chi_{H}$, we may consider mainly the free volume or equation of state contribution. This gives quite generally ${ }^{40} \mathrm{~d} \chi_{H} / \mathrm{d} \phi_{2} \propto \mathrm{d}^{2} \tilde{C}_{p} / \mathrm{d} \tilde{T}$ which must be positive. Another source of the concentration dependence comes from the surface/volume ratio of the polymer relative to that of the solvent ${ }^{2 a}$. In the PIB- $n$-pentane case this ratio ${ }^{2 \mathrm{e}}$ is considerably less than 1 , and leads to a further increase in the predicted $\chi_{H}$ with concentration. The concentration dependence of $\chi_{H}$ parameter appears to indicate a failure of the general equation 8 and possibly a departure of the liquids from corresponding states behaviour. So far, however, no general picture has emerged from these and other discrepancies between theory and experiment. They are of minor importance compared with the qualitative success.

\section{Pressure dependence of the $\chi$ parameter}

Expressions for the contributions to the $\chi, \chi_{H}, \chi_{S}$ parameters at low concentration of polymer may be obtained from equation 8 by developing $\widetilde{G}(\widetilde{T})$ and $(\partial \widetilde{G} / \partial \widetilde{T})$ for the solution in Taylor series in powers of $\left(\widetilde{T}-\widetilde{T}_{1}\right)$. Keeping only second order differences in reduction parameters and also putting $X_{2} \simeq \phi_{2}$, one has ${ }^{40}$ for $\chi_{1}$

$$
\chi_{1}=\frac{-U_{1}(P, T)}{R T}\left(\begin{array}{c}
X_{12} \\
P_{1}^{*}
\end{array}\right)+\frac{C_{p, 1}(P, T)}{2 R} \tau^{2}
$$

where $U_{1}$ and $C_{p .1}$ are the configurational energy and heat capacity of the pure solvent, and $\tau=1-T_{1}^{*} / T_{2}^{*}$. Expressions for the other parameters may be obtained similarly from the coefficients in Table 1 . The theoretical critical lines in Figure 4 are obtained from theor ${ }^{20}$ essentially through the pressure dependence of the $\chi$-parameter. Using the Flory model of the liquid, the pressure dependence of $U_{1}$ and $C_{p, 1}$ are obtained. The $\chi_{12^{-}}$ parameter for the polystyrene-acetone system was fitted ${ }^{19}$ by requiring that the parameters have the critical value at the minimum of the $P(T)$ curve in Figure 4 for the $20400 \mathrm{MW}$ fraction. The remainder of the curve and the curves for the other molecular weights were then predicted. Qualitative agreement with the experimental results is seen in Figure 4. With increasing molecular weight the theoretical curves are displaced to higher $P$ and $T$. The same evolution is observed if $X_{12}$ is increased. As expected from experience with non-polymeric systems ${ }^{21}$, with sufficient increase of $X_{12}$ the minimum in the critical line disappears and $(\mathrm{d} P / \mathrm{d} T)_{c}$ is negative throughout.

\section{Role of size in reduction parameters for the solution}

Equation 8 is based on the assumption that the configurational properties of the solution $\widetilde{G}(\widetilde{T}), \widetilde{H}(\widetilde{T}), \hat{V}(\widetilde{T})$ have the same dependences as the pure components, but with different reduction parameters. These are obtained 


\section{PATTERSON}

through combining rules from the reduction parameters of the pure components. Theories of mixtures of monomeric liquids have emphasized the importance of the combining rules rather than the form of $\widetilde{G}(\widetilde{T}) . \tilde{H}(\tilde{T})$. $\tilde{V}(\tilde{T})$. One of the advantages of the Flory theory has been the omission of any effect in the solution parameters of differences of size of the segments of the components, e.g. in the $X_{12}$ parameter. The Prigogine Average Potential Model ascribed a large effect to this size difference. It is now clear ${ }^{9}$ that the effect was much exaggerated. Nevertheless in recent work ${ }^{9}$ on mixtures of spherical molecules of different diameter, the size difference plays a part in the formulation of the reduction parameters for the solution and leads to negative values of $V^{E}$ and $G^{E}$. One would expect that a difference of chain-diameters would play the role of the difference of diameters of spherical molecules. Then for $G^{E}$ the new effect would add to the combinatorial $G^{E}$ given by the Flory-Huggins equation. However. for spherical molecules of different size. it is found ${ }^{9}$ that the negative $G^{E}$ are approximately reproduced by the Flory-Huggins value of $G^{E}$. There might thus be an overlap between the Flory-Huggins $G^{E}$ and the new size effect for chains of different diameter. Again an examination of the combinatorial entropy of mixing chains of different diameter seems in order. However, the bridge from monomeric mixtures to polymer solutions seems harder to construct than for the free volume effects. It appears that this, and the combinatorial entropy of mixing chains of different diameter. could be the most important topics for further consideration. Finally, it is important to explore the thermodynamic effects of orientational order in the polymer, as apparently revealed by strain birefringence and optical anisotropy measurements ${ }^{4 !}$.

\section{REFERENCES}

'1. Prigogine (with the collaboration of V. Mathot and A. Bellemans). The Molecular Theory' of Solutions, ch. 17. North-Holland Publishing Co.. Amsterdam (1957).

¿"P. J. Flory, Disc. Faruday Soc. 49.7(1970):

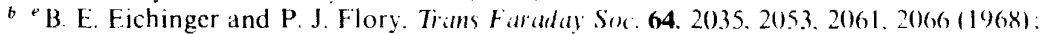

${ }^{f}$ B. F. Fichinger and P. J. Flory, Macromolecules 1. 279 (1968).

${ }^{3}$ V. Mathot. Compt rend. réunion sur les changements de phase's. p. 95. Paris (1952).

${ }^{4}$ R. L. Scott. J. Chem. Phys. 25. 193 (1956).

${ }^{5}$ W. B. Brown. Phil. Trans. Roy. Soc. A250. 175. 221 (1957).

"D. Patterson. Macromolecules 2. 672(1969).

J. S. Rowlinson. Liquids and Liquid Mixture's p. 214. Butterworths. London. 2nd ed. (1969).

${ }^{8}$ R. L. Scott and P. H. van Konynenburg. Disc. Faraday Soc. 49. 87 (1970).

${ }^{9}$ J. S. Rowlinson. Disc. Faraday Soc. 49. 30(1970)

11' D. Patterson. Disc. Faraday Soce. 49, $170(1970)$.

11 R. L. Scott. Disc. Furalay Soc. 49.171 (1970).

12 J. P. Kuenen and W. (i. Robson. Phil. Mag. 48. $180(1899)$ : see also rel. 7. p. 211.

${ }^{13}$ K. S. Siow. G. Delmas and D. Patterson. Macromolecule 5. 29(1972).

14 A. R. Shultz and P. J. Flory. J.Am. Chem. Soc. 74, 4760)(1952).

15 B. A. Wolf. J. W. Breitenbach and H. Senftl. I. Polymer Sci. C. 31. 345 (1970).

if P. Ehrlich and J. J. Kurpen. I. Polymer Sci. A. 1. 3217 (1963).

i. (j. Allen and C. H. Baker. Polymer 6. 181 (1965).

in C. D. Myrat and J. S. Rowlinson. Polymer 6. 645 (1965).

${ }^{19 a}$ L. Zeman, J. Biros, G. Delmas and D. Patterson, J. Phys. (hem. 76, April 1.3 issue (1972).

${ }^{h}$ L. Zeman and D. Patterson, J. Phvs. Chem. 76, April 13 issue (1972).

20) D. Patterson and (i. Delmas. Tran Faraday Soc: 65, 708 (1969).

${ }^{21}$ (j. M. Schneider. Adrum. Ch'm. Phvs. 16. 1 (1970). 
22 D. Oeder and G. M. Schneider. Ber. Bunsenges. Phys. Chem. 73. 229 (1969).

${ }^{23 a}$ G. V. Schulz and M. Lechner. J. Polymer Sci. A-2. 8 (1970):

${ }^{h}$ M. Lechner and G. V. Schulz, European Polymer J. 6. 945 (1970):

'G. V. Schulz and M. Lechner in M. B. Huglin (Ed.). Light Scattering from Polymer Solutions. Academic Press. New York (1971).

24 D. Gaeckle and D. Patterson, Macromolecules 5, April issue (1972).

${ }^{25}$ M. Kurata and W'. H. Stockmayer, Fortschr. Hochpolymer. Forsch. 3, 196 (1963).

26. A. Dondos and H. Benoit. Macromolecules 4. 279 (1971).

27 E. A. Guggenheim. Mixtures. pp. 10-11. Oxford University Press (1952).

${ }^{28}$ S. N. Bhattacharyya. D. Patterson and T. Somcynsky, Physica. 30. 1276 (1964).

29 D. Patterson. Y. B. Tewari. H. P. Schreiber and J. E. Guillet. Macromolecules 4. 356 (1971).

${ }^{30}$ D. H. Everett and R. J. Munn. Trans. Faraday Soc. 60. 1951 (1964).

${ }^{31}$ H. C. Longuet-Higgins. Disc. Faraday Soc. 15. 73 (1953).

${ }^{32}$ M. L. McGlashan. K. W. Morcom and A. G. Williamson, Trans. Faraday Soc. 57, 601 (1961).

${ }^{33}$ H. Tompa. Polymer Solutions. ch. 4. Butterworths. London (1956).

${ }^{34}$ M. Kurata, Ann. N.Y. Acad. Sci. 89, 635 (1961).

${ }^{35 a}$ A. Bellemans and J. van Craen, in Int. Conf. Calorimetry and Thermodynamics, Warsaw (1969).

${ }^{b}$ J. van Craen. Physica. 49, 558 (1970).

${ }^{36}$ D. Patterson and G. Delmas, Disc. Faraday Soc. 49. 98 (1970).

37 W. P. Kao and R. E. Chahal. Unpublished results.

${ }^{38}$ S. Morimoto. J. Polymer Sci. A-1. 6, 1547 (1968).

${ }^{30}$ G. Lewis and A. F. Johnson, J. Chem. Soc. A. 1816 (1969).

40 D. Patterson. J. Polymer Sci. C. 16. 3379 (1968).

41 See for example T. Ishikawa and K. Nagai, Polymer J. 1. 116 (1970); P. Bothorel, J. Colloid Interface Sci. 27, 529 (1968). 